\title{
AEROELASTIC ANALYSIS CONSIDERING STRUCTURAL UNCERTAINTY
}

\section{T. Ueda}

\section{Department of Aerospace Engineering, Nagoya University, Nagoya, JAPAN 464-8603. E-mail: ueda@nagoya-u.jp} Received 1412 2004, accepted 17032005

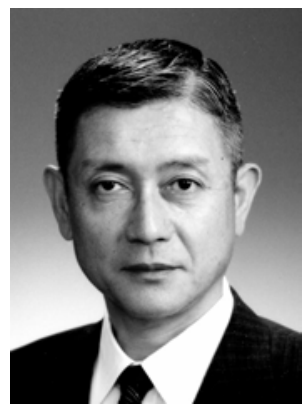

Tetsuhiko UEDA, Prof.

Education:B Sc(Eng.) - 1971, M Sc-1973, D Sc-1976, Aeronautics, University of Tokyo.

Affiliation and functions: 1976-1980 -Researcher, National Aerospace Laboratory, Japan; 1982-1988 -Principal Researcher, NAL; 1980-1982 -Visiting Scholar, Princeton University; 1988-1989 - Invited Scientist, NRC Canada; 1989-1998 -Head, Flight Load Laboratory, NAL; 1998-2001 - Director, Structures Division, NAL; 2001-2003 Director, Structures and Materials Research Center, NAL; 2003-2004 - Director, Aviation Safety Technology Development Center, Japan Aerospace Exploration Agency; Since 2004 -Professor, Nagoya University.

Professional memberships: 1971- Member of the Japan Society for Aeronautical and Space Sciences; 1997- present Liaison Committee, Mechanics and Structures, The Science Council of Japan; 1980 -Member of American Institute of Aeronautics and Astronautics, Associate Fellow.

Teaching: Strength of materials, structural analysis method, structural vibration theory, aero elasticity, general lectures on aeronautics.

Research interests: Flutter, vibrations, fatigue, advanced structural materials, aircraft design.

Present position: Professor of Department of Aerospace Engineering, Nagoya University, Lecturer at Tokyo University of Science, Scientific Consultant of Japan Aerospace Exploration Agency.

\begin{abstract}
Uncertainties in aero elastic analysis are investigated. In aero elastic analysis, we usually find divergence speed or flutter speed by using deterministic equations of motion for elastic wings of aircraft. If any parameter in these equations is sensitive to the critical speed, we should treat it carefully since inaccuracy is inevitable in the production process. Tragic failure may occur if the margin is small. Therefore, it is important to know in the analysis the effects of uncertainties of the critical values when they play a critical role in the design. The sensitivity of parameters in aero elastic analysis is not simple even in linear analysis. It is not always possible to have an analytic form of the sensitivity of uncertainty. In order to evaluate the structural sensitivity of aero elastic phenomena, we have to resort to numerical calculations with uncertain parameters having some random deviations, i.e. the so-called Monte Carlo simulation. In the present study, the divergence speed and flutter speed of a typical wing section with structural uncertainties are discussed, and some results of calculations with scattering parameters in Gaussian distribution will be presented.
\end{abstract}

Key words: flutter analysis, uncertainty, sensitivity analysis, Gaussian distribution.

\section{Introduction}

In the engineering analysis, uncertainty was firstly introduced in an aircraft landing response after the touch down by assuming the external forces from the runway as random load $[4,5]$. The most typical analysis in the aeronautical field including randomness is the gust response of aircraft. They assumes random gust instead of the discrete one that has a fixed mode shape [10]. Recently, the author of this paper has studied an estimation method to find out the condition of in-flight breakups or in-flight collisions of aircraft from the location of structural fragments on the ground [11]. He introduced uncertainties for each fragment falling down through the sky. The uncertainty is included in the aerodynamic forces while falling. The conditions of speed, wind direction, wind speed, and the height of the occurrence were estimated approximately to confirm a scenario for the accidents. There is a good textbook for the stochastic analysis of structure [8]. Owing to the development of the finite element method, the capability of the structural analysis has been broadened to consider the uncertainty in the analytical models. The uncertainty is also practically included in the control theory. In the analysis, sensor noise and disturbance were assumed as white Gaussian distribution in many cases. The method is applied to the aeroservoelastic problem of flutter to be suppressed by the control $[6,2]$. As for the uncertainty materials, fiber directions in a laminated composite were studied for the effects on panel flutter [7]. Numerical simulation technique is also utilized with uncertainties. It is applied to the non-linear problem of limit cycle oscillation [9]. For the typical type of bending-torsion flutter of aircraft wings, the effect of structural uncertainties has not been studied yet. It may be due to the difficulty of the analysis to obtain the flutter boundary. It can easily be thought that a small uncertainty in the structural damping can significantly affect the flutter critical speed when the flutter is in so-called hump mode. Therefore, it is important to see the effects of uncertainty with the structural parameter on the flutter boundary. 


\section{Effects of uncertainty on a divergence speed}

Let us consider a typical section of a wing depicted in Figure 1.

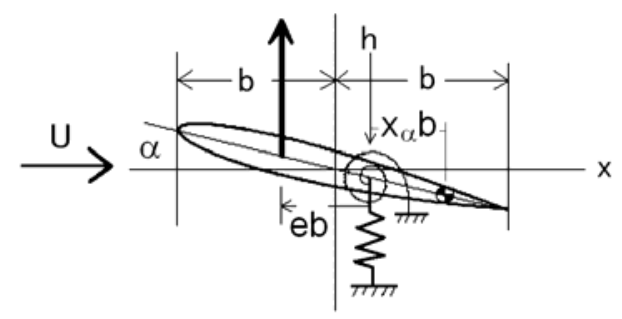

Fig 1. Typical wing section

The divergence speed of this wing section can be given by the formula [10]

$$
U_{D}=\sqrt{\frac{2 K_{\alpha}}{\rho e C_{L \alpha} S}}
$$

where $\rho, C_{L \alpha}, S$, and $K_{\alpha}$ are air density, lift curve slope, wing area, and torsional stiffness, respectively. As depicted in Figure 1, the symbol $e$ denotes the nondimensional distance between aerodynamic center and the elastic axis. Once the divergence speed is given in an analytical form, it is easy to find the sensitivity of each parameter involved in the equation. These sensitivities are closely related to the effects of uncertainty, since it corresponds to the amount of the effects of derivation from a nominal state. The sensitivity of the torsional stiffness to the divergence speed can be obtained by differentiating equation (1) with respect to $K_{\alpha}$ as

$$
\frac{\partial U_{D}}{\partial K_{\alpha}}=\sqrt{\frac{2}{\rho e C_{L \alpha} S}} \frac{1}{2} K_{\alpha}^{-\frac{1}{2}}=\frac{1}{K_{\alpha}} U_{D}
$$

and with respect to $e$ as

$$
\frac{\partial U_{D}}{\partial e}=\sqrt{\frac{2 K_{\alpha}}{\rho C_{L \alpha} S}}\left(-\frac{1}{2}\right) e^{-\frac{3}{2}}=-\frac{1}{e} U_{D}
$$

Similar to the equations above, the effect of uncertainty of each parameter can be evaluated as follows.

$$
\begin{aligned}
& \Delta U_{D}=\frac{\partial U_{D}}{\partial K_{\alpha}} \Delta K_{\alpha}+\frac{\partial U_{D}}{\partial e} \Delta e+\frac{\partial U_{D}}{\partial C_{L \alpha}} \Delta C_{L \alpha}+\frac{\partial U_{D}}{\partial S} \Delta S \\
& =\frac{1}{2}\left[\frac{\Delta K_{\alpha}}{K_{\alpha}}-\frac{\Delta e}{e}-\frac{\Delta C_{L \alpha}}{C_{L \alpha}}-\frac{\Delta S}{S}\right] U_{D}
\end{aligned}
$$

It can be said from equation (4) that the rate of the effects are the same as each parameter with different direction depending on the sign of the derivatives. It should be noted, however, that the second term may cause large deviation when the numerator is small, which means the aeroelastic axis approaches the aerodynamic center.

It can be said from equation (4) that the rate of the effects are the same as each parameter with different direction depending on the sign of the derivatives. It should be noted, however, that the second term may cause large deviation when the numerator is small, which means the aeroelastic axis approaches the aerodynamic center.

\section{Effects of uncertainty on flutter speeds}

Generally, it is difficult to obtain analytical expression of the flutter boundary even for the simple typical section discussed in the previous section. One empirical formula is available for the small frequency ratio of $\omega_{h} / \omega_{\alpha}<<1 \quad[10]$.

$$
\frac{U_{F}}{b \omega_{\alpha}} \cong\left[\frac{m}{\pi \rho b} \frac{r_{\alpha}^{2}}{b+2 X}\right]^{\frac{1}{2}}, r_{\alpha}=\sqrt{\frac{I_{\alpha}}{m b^{2}}}, \omega_{\alpha}=\sqrt{\frac{K_{\alpha}}{I_{\alpha}}}
$$

In the formula above, the parameters $m, I_{\alpha}$, and $r_{\alpha}$ are the mass, moment of inertia, and the radius of gyration of the airfoil, and the variable $\mathrm{X}$ is the dimensional location of the center of gravity.

The flutter dynamic pressure becomes

$$
q_{F}=\frac{1}{2 \pi b} \frac{K_{\alpha}}{(b+2 X)}
$$

The sensitivity of parameters to this critical dynamic pressure can be obtained by differentiating with respect to each parameter as

$$
\begin{aligned}
& \frac{\partial q_{F}}{\partial K_{\alpha}}=\frac{1}{2 \pi b(b+2 X)}=\frac{1}{K_{\alpha}} q_{F} \\
& \frac{\partial q_{F}}{\partial X}=\frac{K_{\alpha}(-2)}{2 \pi b(b+2 X)^{2}}=-\frac{2}{b+2 X} q_{F} \\
& \frac{\partial q_{F}}{\partial b}=-\frac{K_{\alpha}(2 b+2 X)}{2 \pi b\left(b^{2}+2 b X\right)^{2}}=-\frac{2(b+X)}{b(b+2 X)} q_{F}
\end{aligned}
$$

Thus, the effects of uncertainty with these parameters on the flutter dynamic pressure can be written as

$$
\begin{aligned}
\Delta q_{F} & =\frac{\partial q_{F}}{\partial K_{\alpha}} \Delta K_{\alpha}+\frac{\partial q_{F}}{\partial X} \Delta X+\frac{\partial q_{F}}{\partial b} \Delta b \\
& =\left[\frac{\Delta K_{\alpha}}{K_{\alpha}}-\frac{2 \Delta X}{b+2 X}-\frac{2(b+X)}{b(b+2 X)} \Delta b\right] q_{F}
\end{aligned}
$$

It can be said from the equation above that the location of the center of gravity becomes very sensitive against the flutter critical values when the quantity $b+2 \mathrm{X}$ is small.

Let us consider more general flutter boundaries for this typical wing section. The equilibrium equations of this two-degree-of-freedom system become [11]

$$
\begin{aligned}
& m \ddot{h}+S_{\alpha} \ddot{\alpha}+m \omega_{h}^{2} h=Q_{h} \\
& S_{\alpha} \ddot{h}+I_{\alpha} \ddot{\alpha}+I_{\alpha} \omega_{\alpha}^{2} \alpha=Q_{\alpha}
\end{aligned}
$$

where the symbol

$$
S_{\alpha}=m b x_{\alpha}
$$


denotes the static unbalance and the right hand sides of the equations are the external aerodynamic forces for heaving and pitching motion.

The equilibrium equations yield a non-dimensional characteristic equation if we use the static aerodynamic forces as

$$
\left|\begin{array}{cc}
\lambda-R & x_{\alpha} \lambda-Q \\
x_{\alpha} \lambda & r_{\alpha}^{2}(\lambda-1)+e Q
\end{array}\right|=0
$$
defined.

where the following dimensionless parameters are

$$
\frac{\omega_{\alpha}^{2}}{\omega^{2}}=\frac{1}{\lambda}, \quad \frac{q(2 b)}{\omega_{\alpha}^{2} m b} \frac{\partial C_{l}}{\partial \alpha}=Q, \quad \frac{\omega_{h}^{2}}{\omega_{\alpha}^{2}}=R
$$

The determinant equation (14) reduces to the form,

$$
A \lambda^{2}+B \lambda+C=0
$$

Stability boundary can be derived from the discriminant of this quadratic form for $\lambda$.

$$
B^{2}-4 A C=0
$$

This equation is also the quadratic equation for the non-dimensional dynamic pressure parameter of $\mathrm{Q}$. Solving Equation (17) for Q, we obtain the following flutter boundary.

$$
Q_{F}=\frac{E-\sqrt{E^{2}-4 D F}}{2 D}
$$
by

where the quantities in the right hand side are given

$$
\begin{aligned}
& D=\left(e+x_{\alpha}\right)^{2} \\
& E=2 r_{\alpha}^{2}(R+1)\left(e+x_{\alpha}\right)-4 e R\left(r_{\alpha}^{2}-x_{\alpha}^{2}\right) \\
& F=r_{\alpha}^{4}(R+1)^{2}-4\left(r_{\alpha}^{2}-x_{\alpha}^{2}\right) r_{\alpha}^{2} R
\end{aligned}
$$

When we use the critical speed instead of the dynamic pressure, the non-dimensional flutter speed can be related to the dynamic pressure parameter $\mathrm{Q}$ as

$$
\frac{U_{F}}{\sqrt{\mu} \omega_{\alpha} b}=\sqrt{Q_{F}}
$$

where $\mu$ is the mass ratio defined by

$$
\mu=\frac{m}{\pi \rho b^{2}}
$$

\section{Numerical examples}

First, the results of the deterministic solution of Equation (18) is shown in Figure 2 with the parameters $e, x_{\alpha}$, and $r_{\alpha}^{2}$ taken as $0.2,0.2$, and 0.25 , respectively.

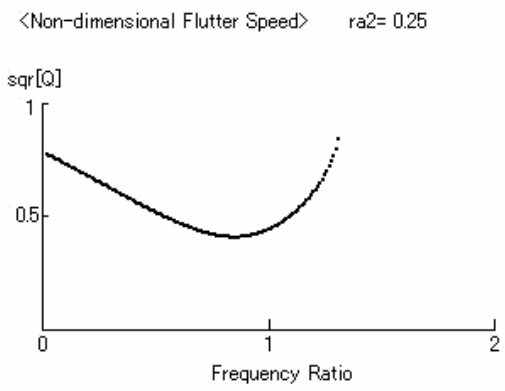

Fig 2. Deterministic flutter boundary

It shows the dependency of the flutter speed on the frequency ratio $\mathrm{R}$. The upper region above the boundary corresponds to the unstable area, i.e. flutter. The boundary becomes the lowest just before one as the frequency ratio increases. The result is reasonable if you think of the coupling between the two degrees of freedom to cause flutter. It may be interesting to compare these results with those of equation (5) for the low frequency ratio. The substitution of the condition, $\mathrm{R}=0$ into equations from (19) to (21) yields

$$
Q_{F}=r_{\alpha}^{2} /\left(e+x_{\alpha}\right)
$$

Using the equations (22) and (23), we can see the relationship between the empirical formula (5) and Equation (24). The comparison leads to a conclusion that the empirical formula corresponds to Equation (5) if the following relationship holds.

$$
C_{l \alpha}=\sqrt{2} \pi
$$

The theoretical value of the lift curve slope of a 2-D airfoil is $2 \pi$, whereas it is $\sqrt{2} \pi$ in equation (25). It is reasonable to have this reduction in the lift curve slope for the empirical formula. In other words, it may be recommended to use $1.4 \pi$ for equation (18) when it is compared with the experiments.

Second, the effect of structural uncertainty is examined by probabilistic simulation of numerical computation. The uncertainty is assumed to be included in the structural parameters with the Gaussian distribution.

We assume the uncertainty of $5 \%$ of standard deviation in the parameters of location of the center of gravity, $x_{\alpha}$. Then the result becomes as illustrated in Figure 3 . The scattering dots are the results of calculation of ten times in each frequency ratio with the step of 0.01 . As can be seen in the figure, the boundary has higher sensitivity for the lower frequency ratio. The deviation in the location of the center of gravity does not have much effect when the frequency ratio is above one.

Next, the effects of uncertainty in the location of the elastic axis are shown in Figure 4 with 5\% standard deviation of the distance, $x_{\alpha}$. 


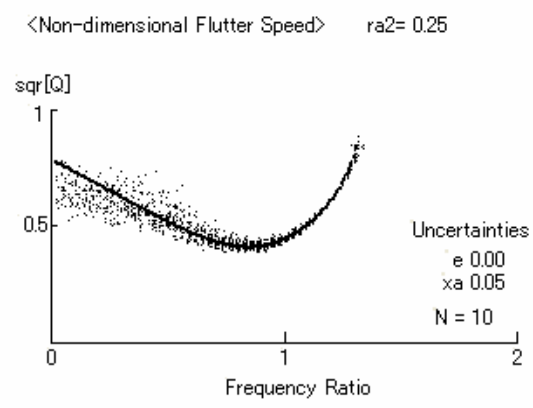

Fig 3. Boundary with uncertainty in the location of center of gravity

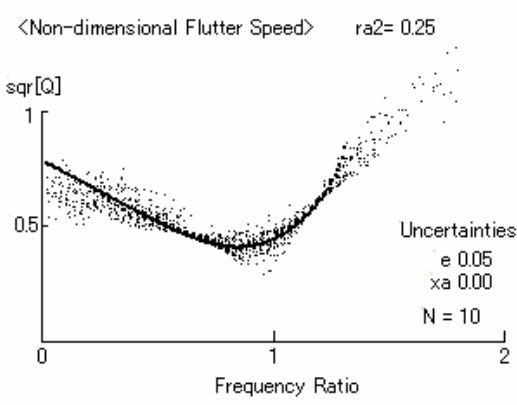

Fig 4. Boundary with uncertainty in the location of the elastic axis

The effects are not monotonous depending on the frequency ratio. It looks very sensitive around the frequency ratio one. On the other hand, there is an insensitive region around the frequency ratio 0.7 . The results imply that the distance between the elastic axis and aerodynamic center is a critical parameter if the frequencies of heaving and torsion modes are almost the same. Figures 5 and 6 shows the combined effects of the uncertainties in the location of elastic axis and in the center of gravity with $5 \%$ and $10 \%$ of the standard deviations. It can be said from these results that the design parameter should be avoided at about the frequency ratio one and should be carefully determined for the low frequency ratio. The results imply that any shift of the center of gravity or the position of the elastic axis in either direction always has a lowering effect on the flutter boundary at the very low frequency ratio.

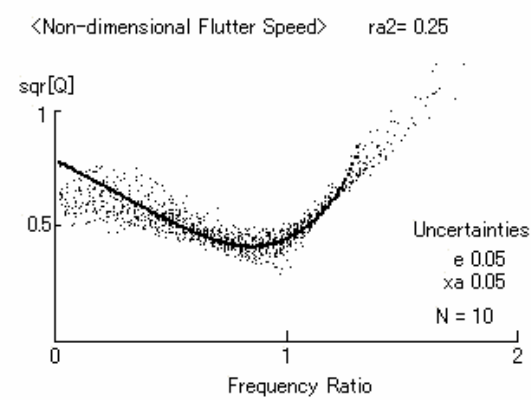

Fig 5. Boundary with uncertainty of $5 \%$ in both parameters

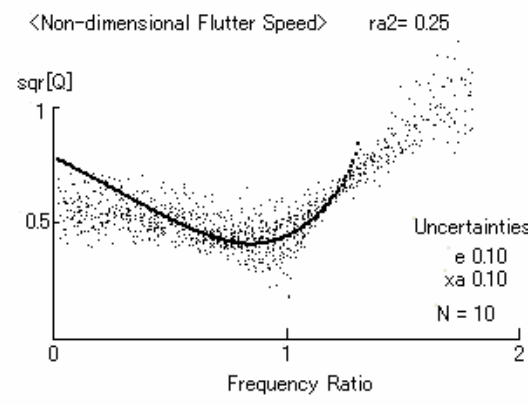

Fig 6. Boundary with uncertainty of $10 \%$ in both parameters

Equation (14) to obtain the flutter boundary also has the parameter of the radius of gyration. To see its influence, the boundary is calculated for the radius of gyration with $60 \%$ of semi-chord length as shown in Figure 7.

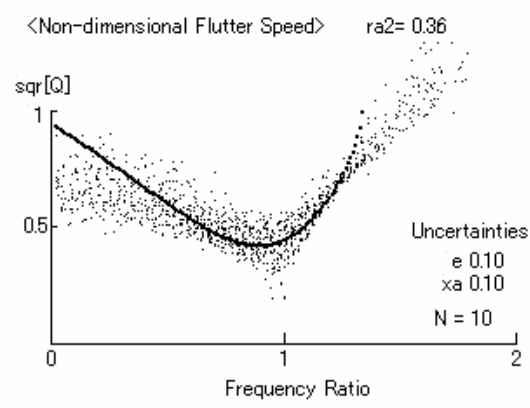

Fig 7. Boundary with a different radius of gyration

The structural uncertainty was assumed to be $10 \%$ for $e$ and $x_{\alpha}$. As can be seen in the figure, the dependency of flutter boundary on the frequency ratio increases in comparison with the previous case where the radius of gyration was $50 \%$ of semi-chord length, i.e. $r_{\alpha}^{2}=0.25$. It should be noted that the uncertainty of the locations of the elastic axis and the center of gravity has lowering effects on flutter boundary although the deterministic boundary increases in the range of the low frequency ratio.

\section{Conclusions}

The effects of structural uncertainty on aero elastic analysis have been studied on the 2-D system of a typical wing section.

For the divergence speed, the effect of uncertainty can be shown analytically.

For the uncertainty of flutter speed, the effect is evaluated with simulations. The sensitivity of the uncertainty is examined numerically by assuming the uncertainty as Gaussian distribution. The results have revealed that the effect is not simple even in the present linear analysis using static aerodynamic forces. This Monte Carlo simulation clearly shows the variation of the sensitivity corresponding to the effects of uncertainty.

In comparison with the empirical formula, it was found that the formula for low frequency ratios coincides 
to the present analysis if the lift curve slope assumes $\sqrt{2} \pi$ instead of the theoretical value of $2 \pi$.

Further study should be made on uncertainties in more practical applications.

\section{References}

1. Bisplinghoff R.L. Ashley H., Halfman R.L. Aeroelasticity. - Cambridge: Addison-Wesley Pub. Co., Inc., 1955.

2. Borglund D., Nilsson U. Robust Wing Flutter Suppression Considering Aerodynamic Uncertainty // Journal of Aircraft. - 2003. - Vol 41, No 2. - P. 331334.

3. Dowell E. H. Curtiss, H.C. Jr. Scanlan R.H. and Sisto F. A modern course in aeroelasticity. - Alphen aan den Rijin: Sijthoff \& Noordhoff, 1978.

4. Fung Y.C. Statistical Aspect of Dynamic Loads // Journal of Aeronanutical Sciences. - 1953. - No 20. P. 317-330.

5. 2. Fung Y.C. The Analysis of Dynamic Stresses in Aircraft Structures during Landing as Nonstationary Random Processes // Journal of Applied Mechanics. 1955. - P. 449-457.
6. Kerpel M., Moulin B., Idan M. Robust Aeroservoelastic Design with Structural Variations and Modeling Uncertainties // Journal of Aircraft. 2003. - Vol 40, No 5. - P. 946-960.

7. Liaw D.G., Yang H.T.Y. Relability of Initially Compressed Uncertain Laminated Plates in Supersonic Flow // AIAA Journal. - 1991. - Vol 29, No 6. - P. 952-960.

8. LinY.K. Probabilistic Theory of Structural Dynamics. - New York: McGraw-Hill, Inc., 1967.

9. Pettit C.L. and Beran P.S. Effects of Parametric Uncertainty on Airfoil Limit Cycle Oscillation // Journal of Aircraft. - 2003. - Vol 40, No 5. - P. 1004-1006.

10. Pototsky A.S., Zeiler, T.A., Perry B.III. Calculation Time-Correlated Gust Loads Using Matched Filter and Random Process Theories // Journal of Aircraft. 1991. - Vol 28, No 5. - P. 346-352.

11. Ueda T., Kanda A., Wataki A. Scattering Analysis for Wreckage of In-Flight Breakup // $22^{\text {nd }}$ ICAS Congress, Harrogate, England. - 2000. - ICAS-662. P. 1-6. 